\title{
Investigating Preoperative Hematologic Markers for Prediction of Ovarian Cancer Surgical Outcome
}

\author{
Tahereh Ashrafganjoei ${ }^{1}$, Mahdiss Mohamadianamiri' ${ }^{2 *}$, Farah Farzaneh ${ }^{1}$, \\ Maryam Sadat Hosseini ${ }^{3}$, Maliheh Arab ${ }^{3}$
}

\begin{abstract}
Purpose: The current study aimed at assessing the association between neutrophil-lymphocyte ratio (NLR) and platelet lymphocyte ratio (PLR) for the prognosis of the surgical outcome of epithelial ovarian cancer (EOC). Materials and Methods: EOC patient medical records of surgical operations between January, 2005 and December, 2015 were reviewed and their data of clinicopathological complete blood counts (CBCs) and surgical outcomes were collected. To assess their effects on surgical outcomes, PLR and NLR optimal predictive values were determined and then compared with each other. Results: A statistically significant relation was found between surgical outcomes and NLR and PLR ( $p<0.001$ and $p<0.001$ ), for which new cutoff points were gained (PLR: 192,3,293; NLR: 3). The sensitivity and specificity were 0.74 and 0.67 , respectively for PLR and 0.74 and 0.58, for NLR. Conclusions: NLR and PLR seem to be useful methods for the prediction of surgical outcomes in patients with EOCs. Increased NLR and PLR proved to be beneficial for poor surgical outcomes. Moreover, PLR increase showed further help in the predicting outcome of EOC suboptimal debulking.
\end{abstract}

Keywords: Neutrophil-lymphocyte ratio - platelet-lymphocyte ratio - ovarian masses - optimal debulking - outcome

Asian Pac J Cancer Prev, 17 (3), 1445-1448

\section{Introduction}

From among gynecologic cancers, epithelial ovarian cancer (EOC) is the major cause of mortalities in the United States, accounting for $3.6 \%$ of all types of gynecologic cancers (3rd rank) throughout the world (Jemal et al., 2010), while less than $40 \%$ of woman afflicted with ovarian cancer are treated (Siegel et al., 2015). A standard management for EOC patients involves a primary cytoreductive surgery followed by adjuvant chemotherapy if needed (Fader and Rose, 2007). Still, a poor overall prognosis is rehearsed for patients with advanced EOC despite improved surgical techniques and effectively developed chemotherapy (Markman et al., 2001; Fader and Rose, 2007). A major reason for this poor treatment is that most EOC patients are frequently involved in an advanced stage of the disease. Furthermore, other characteristics such as, race, age, histologic type, grade, tumor marker, and residual tumor after surgery are required to be identified as prognostic factors (Fathalla, 1972; Vergote et al., 1993; Kodama et al., 1997).

Nevertheless, no professional societies exist to recommend routine screening for ovarian cancer among the general population at present (Clarke-Pearson, 2009). Helpful information on the possible clinical outcomes of cancer patients is provided through prognostic factors by which the patients can be classified into different risk groups. Thus, it is vital to be able to access reliable prognostic markers when designing treatment plans to discuss with the patients since clinical management decisions are often challenging (Rochet et al., 2012). Diagnostic sensitivity can be improved via hematological, immunological, and inflammatory markers studied through CA-125 advanced technology. In addition, it is suggested to use some parameters of CBC panel in the diagnosis and prognosis of the disease (Yildirim et al., 2014). Clinical outcomes are strongly affected by the immune response of the host to ovarian cancer (Milne et al., 2012). Neutrophil and the lymphocyte counts were found to increase and relatively decrease as a result of the systemic inflammatory response, respectively. Moreover, a correlation was seen between thrombocytosis associated with the current tumor aggressiveness and quick prognosis for the relevant high recurrence rate. NLR enhancement of epithelial ovarian cancers was discovered to correlate with adverse clinical outcomes (Kokcu et al., 2014; Yildirim et al., 2014). An advanced stage of EOC led to the inoperability of preoperative thrombocytosis ( $\mathrm{Li}$ et al., 2004). Attempting to improve NLR prognostic function, a high NLR was reported to associate with an adverse OS in numerous solid tumors in few studies. NLR accounts for an inexpensive biomarker readily available (Templeton et al., 2014). An investigation on EOC (Asher et al., 2011) was even indicative of the better diagnostic 
Tahereh Ashrafganjoei et al

performance of PLR than NLR. Nonetheless, some unfavorable clinicopathological features were observed to be associated with an augmentation in neutrophils, platelets, NLR, or PLR in the previous clinical studies on many cancers (Thavaramara et al., 2011).

The purpose of the current research was to assess the effects of PLR and NLR parameters from CBC panel on the prediction of EOC surgical outcome.

\section{Materials and Methods}

Medical record of patients diagnosed with epithelial ovarian cancer who underwent primary staging exploratory laparotomy at Emam Hossein hospital, Department of Gynecology and Obstetrics between January 2005 and December 2015 were reviewed and 200 patients were collected. The study included 168 patients and 32 patients were excluded. The exclusion criteria were: fertility spairing surgery, incomplete CBC before surgery, insufficient medical history, bone marrow suppression, current infection, any medical condition which influences $\mathrm{CBC}$ markers, previous history of local or systemic infection, any medication that is related to patients' inflammatory condition such as corticosteroids and hematologic malignancy. CA-125 was recorded as the primary tumor marker. The cut-off value for CA-125 was $35 \mathrm{IU} / \mathrm{ml}$.

Patients' preoperative data, including demographic features, complete blood count with differentials, tumor histologic type, grade, stage, optimal or suboptimal debulking and serum CA-125 levels were retrospectively analyzed. The relationship between the preoperative values of WBC, platelet number, NLR, PLR and tumor features, including stage and CA-125 levels were evaluated. Optimal surgery was defined when the size of each foci of residual disease after surgery was $<1 \mathrm{~cm}$. The selected CBC is the nearest one to the surgery.

Role based on data from many previous reports $(\mathrm{Li}$ et al., 2004). NLR of 2.6 and PLR of 200 was used as a cut-off level based on finding from our previous study reporting prognostic role of NLR and PLR in EOC patients (Asher et al., 2011; Thavaramara et al., 2011). Cut-off values, sensitivity, specificity, PPD, and NPD values were calculated for all cases. In terms of the results achieved, $\mathrm{p}<0.05$ was statistically considered to be a significant difference between the groups at a confidence interval of $95 \%$.

\section{Statistical analysis}

Categorical variables were represented by count with percentage, and continuous variables were represented by mean with standard deviation. Independent sample t-test, Mann-Whitney test, and chi-squared test were applied to compare groups. The area under the roc curve (AUC), Sensitivity, Specify, positive predictive value (PPV), negative predictive value (NPV), positive likelihood ratio (PLR), and negative likelihood ratio (NLR) were calculated as accuracy measures. Best cut points were obtained by using the optimal sensitivity and specificity values determined by the ROC curves. The AUCs were

Table 1. Characteristics of Patients According to the Surgical Outcome

\begin{tabular}{|c|c|c|c|c|}
\hline \multirow{2}{*}{ Characteristics } & Total & Sub-optimal & Optimal & \multirow{2}{*}{$\mathrm{P}$ value } \\
\hline & $(n=168)$ & $(n=50)$ & $(n=118)$ & \\
\hline Age & $52.1 \pm 12.72$ & $53.5 \pm 11.43$ & $51.5 \pm 13.23$ & 0.36 \\
\hline Stage & & & & $<0.001$ \\
\hline 1 & $31(18.5)$ & $0(0)$ & $31(26.3)$ & \\
\hline 2 & $11(6.5)$ & $0(0)$ & $11(9.3)$ & \\
\hline 3 & $104(61.9)$ & $34(68)$ & $70(59.3)$ & \\
\hline 4 & $22(13.1)$ & $16(32)$ & $6(5.1)$ & \\
\hline Histology & & & & $<0.001$ \\
\hline Endometrioid & $17(10.1)$ & $3(6)$ & 14 (11.9) & \\
\hline Serous & 135 (80.4) & $42(84)$ & $93(78.8)$ & \\
\hline Mucinous & $14(8.3)$ & $5(10)$ & $9(7.6)$ & \\
\hline Transitional & $1(0.6)$ & $0(0)$ & $1(0.8)$ & \\
\hline Clear-cell & $1(0.6)$ & $0(0)$ & $1(0.8)$ & \\
\hline Grade & & & & $<0.001$ \\
\hline 1 & $53(31.5)$ & $9(18)$ & $44(37.3)$ & \\
\hline 2 & $57(33.9)$ & $11(22)$ & $46(39)$ & \\
\hline 3 & $58(34.5)$ & $30(60)$ & $28(23.7)$ & \\
\hline Lymph & $26.4 \pm 9.33$ & $18.9 \pm 7.47$ & $29.6 \pm 8.13$ & $<0.001$ \\
\hline PMN & $67.3 \pm 10.36$ & $75.2 \pm 9.19$ & $63.9 \pm 8.91$ & $<0.001$ \\
\hline CA125 & $285.8 \pm 299.40$ & $353.9 \pm 397.98$ & $256.9 \pm 242.46$ & 0.113 \\
\hline NLR & $3.1 \pm 2.26$ & $4.8 \pm 3.04$ & $2.4 \pm 1.32$ & $<0.001$ \\
\hline PLR & $184.2 \pm 110.42$ & $280.6 \pm 135.07$ & $143.4 \pm 64.11$ & $<0.001$ \\
\hline PLT & $289.8 \pm 92.78$ & $342.8 \pm 93.11$ & $267.3 \pm 83.36$ & $<0.001$ \\
\hline WBC & $8.9 \pm 14.62$ & $7.6 \pm 3.46$ & $9.4 \pm 17.30$ & 0.775 \\
\hline
\end{tabular}

The mean age of patients was 52.1 ranged (23-88). The most common histologic subtypes was serous (80.4\%) and the less common were clear cell $(0.6 \%)$ and transitional $(0.6 \%)$ 
Table 2. Predictive Values for Different Cutoffs of PLR, NLR, and PLT

\begin{tabular}{lccccc}
\hline \multirow{2}{*}{ Cutoff-Point } & \multicolumn{2}{c}{ PLR } & NLR & PLT \\
\cline { 2 - 6 } & 200 & 192 & 2.6 & 3 & 293 \\
\hline Sensitivity & $0.76(0.62-0.87)$ & $\mathrm{v}$ & $0.86(0.73-0.94)$ & $0.76(0.62-0.87)$ & $0.70(0.55-0.82)$ \\
Specificity & $0.83(0.75-0.89)$ & $0.69(0.59-0.77)$ & $0.81(0.72-0.87)$ & $0.70(0.61-0.78)$ & $0.86(0.79-0.92)$ \\
Positive predictive value & $0.70(0.56-0.82)$ & $0.67(0.54-0.79)$ & $0.54(0.42-0.65)$ & $0.62(0.49-0.74)$ & $0.50(0.38-0.62)$ \\
Negative predictive value & $0.89(0.82-0.94)$ & $0.92(0.85-0.96)$ & $0.92(0.84-0.97)$ & $0.89(0.81-0.94)$ & $0.85(0.76-0.91)$ \\
Positive likelihood ratio & $5.61(3.46-9.07)$ & $4.84(3.18-7.36)$ & $2.74(2.05-3.66)$ & $3.90(2.62-5.81)$ & $2.36(1.69-3.29)$ \\
Negative likelihood ratio & $0.28(0.17-0.46)$ & $0.22(0.12-0.39)$ & $0.20(0.10-0.41)$ & $0.30(0.18-0.49)$ & $0.43(0.27-0.66)$ \\
\hline
\end{tabular}

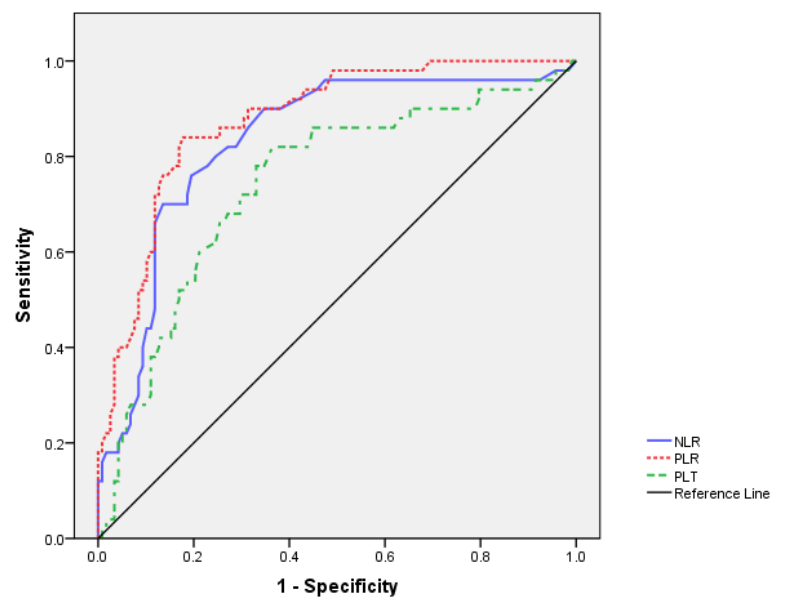

Figure 1. Reciever Operating Characteristics of PLR, NLR, and PLT for Prediction of Surgical Outcome

compared by Delong test and their P values were adjusted by bonferroni correction. We set 0.05 as the level of significance and statistical analysis was performed using R-package version 3.0.1.

\section{Results}

The study included 168 women diagnosed with epithelial ovarian cancer who underwent primary staging exploratory laparotomy. Mean age of patients were 52.1 ranged (23-88), the number of cases according to stages was as follows: stage I: 31 , stage II: 11, stage III: 104, stage IV: 22 . The number of cases according to surgical outcome was optimal $118(70.2 \%)$ and suboptimal 50 (29.8\%) and comparisons were performed between these two groups. Comparison of hematologic parameters between the optimal and suboptimal surgical outcome was shown in Table 1. Among the hematologic parameters, NLR, and PLR were significantly elevated in suboptimal surgical outcome ( $\mathrm{p}<0.001, \mathrm{p}<0.001$ respectively).

Figure1 shows receiver operating characteristic of PLR, NLR, and PLT for prediction of surgical outcome. The area under the roc curves (AUC) for predicting surgical outcome were obtained 0.877 (95\% CI: 0.82$0.92)$ for PLR, 0.836 (95\% CI: 0.77-0.89) for NLR, and 0.742 (95\% CI: 0.67-081) for PLT. Pair-wise comparisons between AUCs of these ratios show that there wasn't any significant difference between prediction validity of PLR and NLR ( $p=0.246)$, but PLR had a better prediction that PLT ( $p<0.001)$. The best cut off points of PLR, NLR, and PLT for predicting surgical outcome was 293, 192, and
3 , respectively. The predictive values of these ratios are represented in table 2 .

\section{Discussion}

Different prognostic factors such as, age, race, stage, grade, cell type, tumor marker, and residual tumor after surgery were reported to predict EOC outcome by various studies (Kodama et al., 1997; Li et al., 2004). Based on the pathologic characteristics of cancer, any prognostic factors, except for age, race, and tumor marker, are capable of being evaluated during or after surgery. Attempting to assess a prognostic role in EOC, many studies have recently included $\mathrm{CBC}$ for a pre-operative laboratory evaluation. Such blood components as neutrophil and platelet counts (Levin and Conley, 1964; Li et al., 2004; Soonthornthum et al., 2007), as well as NLR (Cho et al., 2009) and lately PLR (Asher et al., 2011) have been of a particular interest. As an independent EOC prognostic factor, platelet count showed to have a role in thrombocytosis accompanied with an inoperable more advanced cancer in many early studies (Levin and Conley, 1964; Li et al., 2004; Soonthornthum et al., 2007). Also, the prognostic roles of NLR and PLR were investigated in some other studies, the results of which were incongruent (Cho et al., 2009). Although a significant association of pre-operative NLR $\geq 3$ and suboptimal surgery was found in this research, Cho et al. (Cho et al., 2009) discovered a poor survival outcome was accompanied with NLR $\geq$ 2.6 and enhanced CA125. PLR, NLR and PLT functions were analyzed and compared for the possible EOC surgical outcome in the present investigation. A modest predictive value was found for each of these blood components as well as their ratios (e.g. platelet count, PLR or NLR) for the determination of a residue after surgery. Yet, from among the mentioned indicators, PLR demonstrated to have a more improved function.

Consequently, a poor surgical outcome was seen to significantly associate with NLR $\geq 3$, PLR $\geq 192$, and PLT $\geq 293000$ in the analyses.

Though not reaching a statistical significance in his study on PLR and NLR in 84 pancreatic cancer patients, Bhatti et al. (Bhatti et al., 2010) discovered shorter and poorer survivals were significantly and tendingly associated only with NLR $\geq 4$ and PLR $\geq 200$, respectively (Bhatti et al., 2010). To determine NLR predictive value, a cutoff value of 3 was used and a better function was found with PLR at either 3 or 4 compared to NLR in our study. In another study of NLR and PLR conducted on 324 gastric cancer patients by Wang et al. (Wang 


\section{Tahereh Ashrafganjoei et al}

et al., 2012), a combination of C-reactive protein and albumin as prognostic indicators were used for survival compared to the Glasgow Prognostic Score. Except for the Glasgow Prognostic Score, NLR or PLR were not found to independently associate with an overall disease-free survival (Wang et al., 2012). Nevertheless, PLR was reported to have a significant prognostic role in the study of Smith et al. (Smith et al., 2008), who observed pancreatic cancer patients' poor survivals were associated with PLR $\geq 160$ and a high level of CA19- 9. Compared to an elevation of each of the two CA19-9 and PLR markers, the worst survival outcome was even found with their combination (Smith et al., 2008). Only one of the previous reports of PLR in EOC (Asher et al., 2011) was inconsistent with the significant results of pancreatic cancer obtained by Smith et al. (2008).

On a study on preoperative PLR and NLR in 235 ovarian cancer patients, Asher et al. (2011) discovered a poor survival was significantly accompanied with age, stage, grade, absolute neutrophil count, platelet count, NLR ( $\geq 4)$, PLR ( $\geq 300$ ), and surgical outcome. The only independent prognostic factors for survival were found to be PLR, stage, and residual disease. The different PLR statistical significances faced in all the mentioned studies might be due to the patients' different numbers or proportions of features, such as the disease stage and primary surgical results, thus leading to an unfavorable prognosis for those with high PLR levels. Anyhow, heterogeneity of early and advanced stage patients in the study communities was a common restriction in both Asher et al. (2011) and our studies. The small number of patients was another limitation in our research.

In the current clinical study, it was concluded that a poor prognosis was associated with an enhancement in all blood components in EOC surgery. Compared to NLR or thrombocytosis, PLR revealed to be a better independent prognostic indicator for EOC surgery in a univariable analysis, but not in a multivariable analysis. Thus, to corroborate the prognostic roles of blood components, further research in a larger and more homogeneous population is required.

\section{Acknowledgements}

The authors thank to Department of Obstetrics and Gynecology, Preventative Gynecology Research Center (PGRC), Imam Hossein Hospital, Shahid Beheshti University of Medical Sciences, for their assistance. The authors are also grateful to the Clinical Research Development Unit, Imam Hossein Hospital, Shahid Beheshti University of Medical Sciences, Tehran, Iran.

\section{References}

Asher V, Lee J, Innamaa A, et al (2011). Preoperative platelet lymphocyte ratio as an independent prognostic marker in ovarian cancer. Clin Transl Oncol, 13, 499-503.

Bhatti I, Peacock O, Lloyd G, et al (2010). Preoperative hematologic markers as independent predictors of prognosis in resected pancreatic ductal adenocarcinoma: neutrophillymphocyte versus platelet-lymphocyte ratio. Am J Surg,
200, 197-203.

Cho H, Hur HW, Kim SW, et al (2009). Pre-treatment neutrophil to lymphocyte ratio is elevated in epithelial ovarian cancer and predicts survival after treatment. Cancer Immunol Immunother, 58, 15-23.

Clarke-Pearson DL (2009). Clinical practice. Screening for ovarian cancer. $N$ Engl J Med, 361, 170-7.

Fader AN, Rose PG (2007). Role of surgery in ovarian carcinoma. J Clin Oncol, 25, 2873-83.

Fathalla MF (1972). Factors in the causation and incidence of ovarian cancer. Obstet Gynecol Surv, 27, 751-68.

Jemal A, Siegel R, Xu J, et al (2010). Cancer statistics, 2010.CA Cancer J Clin, 60, 277-300.

Kodama S, Tanaka K, Tokunaga A, et al (1997). Multivariate analysis of prognostic factors in patients with ovarian cancer stage I and II. Int J Gynaecol Obstet, 56, 147-53.

Kokcu A, Kurtoglu E, Celik H, et al (2014). May the platelet to lymphocyte ratio be a prognostic factor for epithelial ovarian cancer? Asian Pac J Cancer Prev, 15, 9781-4.

Levin J, Conley CL (1964). Thrombocytosis Associated with Malignant Disease. Arch Intern Med, 114, 497-500.

Li AJ, Madden AC, Cass I, et al (2004). The prognostic significance of thrombocytosis in epithelial ovarian carcinoma. Gynecol Oncol, 92, 211-4.

Markman M, Bundy BN, Alberts DS, et al (2001). Phase III trial of standard-dose intravenous cisplatin plus paclitaxel versus moderately high-dose carboplatin followed by intravenous paclitaxel and intraperitoneal cisplatin in small-volume stage III ovarian carcinoma: an intergroup study of the gynecologic oncology group, southwestern oncology group, and eastern cooperative oncology group. J Clin Oncol, 19, 1001-7.

Milne K, Alexander C, Webb JR, et al (2012). Absolute lymphocyte count is associated with survival in ovarian cancer independent of tumor-infiltrating lymphocytes. $J$ Transl Med, 10, 33.

Rochet NM, Markovic SN, Porrata LF (2012). The role of complete blood cell count in prognosisâ watch this space! Oncol Hematology Review, 8, 76-82.

Siegel RL, Miller KD, Jemal A (2015). Cancer statistics, 2015. CA Cancer J Clin, 65, 5-29.

Smith RA, Ghaneh P, Sutton R, et al (2008). Prognosis of resected ampullary adenocarcinoma by preoperative serum CA19-9 levels and platelet-lymphocyte ratio. J Gastrointest Surg, 12, 1422-8.

Soonthornthum T, Suraseraneewong V, Kengsakol K, et al (2007). Thrombocytosis in advanced epithelial ovarian cancer. J Med Assoc Thai, 90, 1495-500.

Templeton AJ, McNamara MG, Seruga B, et al (2014). Prognostic role of neutrophil-to-lymphocyte ratio in solid tumors: a systematic review and meta-analysis. J Natl Cancer Inst, 106, 124.

Thavaramara T, Phaloprakarn C, Tangjitgamol S, et al (2011). Role of neutrophil to lymphocyte ratio as a prognostic indicator for epithelial ovarian cancer. J Med Assoc Thai, 94, 871-7.

Vergote IB, Kaern J, Abeler VM, et al (1993). Analysis of prognostic factors in stage I epithelial ovarian carcinoma: importance of degree of differentiation and deoxyribonucleic acid ploidy in predicting relapse. Am J Obstet Gynecol, 169, 40-52.

Wang DS, Ren C, Qiu MZ, et al (2012). Comparison of the prognostic value of various preoperative inflammation-based factors in patients with stage III gastric cancer. Tumour Biol, 33, 749-56.

Yildirim MA, Seckin KD, Togrul C, et al (2014). Roles of neutrophil/lymphocyte and platelet/lymphocyte ratios in the early diagnosis of malignant ovarian masses. Asian Pac J Cancer Prev, 15, 6881-5. 